\section{Rheumatoide Arthritis: Alkoholkonsum hat keine Vorteile}

Baker JF et al. Changes in Alcohol Use and Associations With Disease Activity, Health Status, and Mortality in Rheumatoid Arthritis. Arthritis Care Res (Hoboken) 2020; 72: 301-308. doi: $10.1002 /$ acr.23847

Einige wissenschaftliche Untersuchungen deuten auf einen Zusammenhang zwischen der Rheumatoiden Arthritis und dem Alkoholkonsum hin: Patienten, die in mäßigem Umfang Alkohol zu sich nehmen, haben eine bessere Lebensqualität sowie eine bessere funktionelle Leistungsfähigkeit. Dies liegt allerdings daran, dass im Wesentlichen nur Betroffene mit geringer Krankheitsaktivität Alkohol trinken. Schwer kranke Patienten schränken dagegen ihren Konsum ein.

Zu diesem Ergebnis kommt ein Team von US-Forschern nach Auswertung umfangrei- cher Patientendaten. Sie wollten klären, ob Rheumapatienten hinsichtlich der Krankheitsaktivität sowie des Sterberisikos von einem Alkoholkonsum profitieren, oder ob diesbezüglich eine umgekehrte Kausalität besteht. Ferner suchten sie nach Prädiktoren für eine Veränderung des Trinkverhaltens. Die Forscher analysierten 16762 Patienten mit einer Rheumatoiden Arthritis, die zwischen 1999 und 2016 in die „Forward, The National Databank for Rheumatic Diseases“ aufgenommen worden waren. Alle registrierten Teilnehmer beantworten 2-mal pro Jahr umfangreiche Fragebögen. Unter anderem werden dabei auch detaillierte Informationen zum Alkoholkonsum erhoben und die Krankheitsaktivität wird mithilfe der „Patient Activity Scale-II“ (PAS-II) objektiviert.

\section{Ergebnisse}

Insgesamt werteten die Forscher 121280 Beobachtungen aus. Konsumunterbrechungen waren bei den Alkohol trinkenden Patienten verbreitet. Unabhängige Faktoren, die in diesem Kollektiv für einen Alkoholverzicht prädisponierten, waren höheres Alter, Adipositas, stärkere Komorbiditäten sowie Arbeitsunfähigkeit. Männliches Geschlecht, weiße Hautfarbe, eine bessere physische und psychische Lebensqualität, ein höherer Bildungsstand sowie ein höheres Haushaltseinkommen gingen dagegen mit einer geringeren Wahrscheinlichkeit für Konsumunterbrechungen einher. Einen Konsumbeginn nach einer Alkoholabstinenz beobachteten die Wissenschaftler ebenfalls regelmäßig. Eine geringe Wahrscheinlichkeit für den Konsumbeginn hatten in dieser Patientengruppe ältere und arbeitsunfähige Personen sowie Methotrexat-Anwender. Männliches Geschlecht, eine gute physische und psychische Lebensqualität, ein hoher Bildungsstand sowie ein hohes Haushaltseinkommen begünstigten dagegen einen Trinkbeginn. Alkoholkonsumenten wiesen zwar bei Verwendung traditioneller Berechnungsmodelle einen signifikant geringeren PAS-II-Score sowie eine geringere Mortalität auf, in sogenannten marginalen strukturellen Modellen ließen sich diese Zusammenhänge allerdings nicht nachweisen. Ferner bestand kein Zusammenhang zwischen dem aktiven Trinken, einem kürzlicher Konsumverzicht oder einem kürzlicher Konsumbeginn und der Krankheitsaktivität bzw. dem Versterben. 
FAZIT

Bei hoher Aktivität der Rheumatoiden Arthritis, starken gesundheitlichen Belastungen sowie reduzierter Lebensqualität sinkt der Alkoholkonsum bzw. abstinente Patienten setzen ihren Verzicht fort, schlussfolgern die Autoren. Es besteht also tatsächlich ein - allerdings reverser - Zusammenhang zwischen dem Alkoholkonsum und einer geringeren Krankheitsaktivität. Einen protektiven Alkoholeffekt schließen die Forscher angesichts dieser Ergebnisse aus.

Dr. med. Judith Lorenz, Künzell 